\title{
Computer-based cognitive rehabilitation research in a military treatment facility: Recruitment, compliance, and lessons learned
}

\author{
Katherine W. Sullivan ${ }^{\mathrm{a}, \mathrm{b}, *}$, Nancy Pearl Solomon ${ }^{\mathrm{b}}$, Michael Pramuka ${ }^{\mathrm{a}, \mathrm{b}}$, Julia E. Quinn ${ }^{\mathrm{b}, \mathrm{c}}$, \\ Kathryn A. Teixeira ${ }^{\mathrm{b}, \mathrm{c}}$ and Louis M. French ${ }^{\mathrm{a}, \mathrm{b}, \mathrm{c}, \mathrm{d}}$ \\ ${ }^{a}$ Defense and Veterans Brain Injury Center, Silver Spring, MD, USA \\ ${ }^{\mathrm{b}}$ Walter Reed National Military Medical Center, Bethesda, MD, USA \\ ${ }^{\mathrm{c}}$ Center for Neuroscience and Regenerative Medicine, Bethesda, MD, USA \\ ${ }^{\mathrm{d}}$ Uniformed Services University of the Health Sciences, Bethesda, MD, USA
}

Received 24 June 2013

Accepted 5 February 2014

\begin{abstract}
.
BACKGROUND: Evidence-based approaches to cognitive rehabilitation are limited; however, new technologies such as braintraining computer programs provide opportunities for novel interventions.

OBJECTIVE: This paper describes a randomized controlled training study in a military treatment facility with service members who had combat-related cognitive symptoms. It examines challenges in study design and implementation, and provides "lessons learned" with proposed solutions.

METHODS: Participants were randomly assigned to one of two 6-week computer-based cognitive training (CBCT) programs or a treatment-as-usual (TAU) control group. Feasibility assessments included reasons for consent refusal, compliance, and drop-out rates.

RESULTS: The intended sample size for the study was 114 participants before attrition. Of 291 patients referred over 2.5 years, 120 were eligible, 38 consented to participate, and 18 completed the study. Forty-two percent of the participants assigned to CBCT groups completed the required 30 sessions in 6.5 to 32 weeks. Study-design factors that affected enrollment and compliance included eligibility restrictions, lack of a computer-based control condition, and inflexible scheduling.

CONCLUSIONS: Successful implementation of a high-dose computer-based clinical trial will require design changes such as expanded inclusion criteria, control by sham computer program or wait-list, dosing flexibility, and web-based options.
\end{abstract}

Keywords: Traumatic brain injury, brain training, compliance

\section{Introduction}

There is a need for evidence-based treatment programs for patients with cognitive dysfunction following traumatic brain injury (TBI) in both civilian

*Corresponding author: Katherine Sullivan, Department of Rehabilitation, Walter Reed National Military Medical Center, 8901 Wisconsin Avenue, Bethesda, MD 20889, USA. Tel.: +1 301319 2178; E-mail: Katherine.w.sullivan.ctr@mail.mil. and military populations. Advances in science and technology are providing opportunities for innovative approaches in rehabilitation through computer-based cognitive training (CBCT) programs. Benefits of these programs include high user engagement and a lowcost extension of treatment beyond the clinical setting for tele-health, continued care, and maintenance. Advances in neuroplasticity-based research suggest that highly repetitive, adaptive, novel, and targeted stimuli can enhance brain performance. These novel brain- 
training treatment tools continue to emerge on the commercial market at a steady rate. Evidence is needed for the efficacy and feasibility of implementing these CBCT programs as a treatment method in varied settings and with varied populations. One reason for the paucity of evidence for the treatment of cognitive dysfunction in both traditional cognitive rehabilitation and with computer-based approaches is the challenge of executing well-designed treatment protocols. This paper reports our first attempt to evaluate the effectiveness of two CBCT tools with patients at a military treatment facility (MTF). The study provided limited outcome data yet valuable information regarding the feasibility of using computer programs with military service members during rehabilitation. It also revealed challenges related to conducting such a study. It is the intent of the authors to share lessons learned and offer recommendations that may assist future researchers in their efforts to conduct similar studies with computerbased rehabilitation tools.

\section{Background}

TBI is a major public health concern and has been described as "a signature injury" of the war in Iraq and Afghanistan [1]. A total of 294,172 military service members were diagnosed with a TBI from 2000 through 2013; 80\% were diagnosed specifically with mild TBI (mTBI) [2]. Individuals with TBI commonly report cognitive dysfunction related to their injuries. In some cases, these reported difficulties are related to objectively measured impairments in attention, memory, or other cognitive domains due to the effects of brain injury, side effects of therapeutic interventions, or extra-cranial injuries. In other cases, the complaints reflect emotional distress including mood disorders or combat-induced stress symptoms. Post-concussive symptoms should resolve within weeks to months; however, for reasons not fully understood, many service members who experience a $\mathrm{mTBI}$ in combat have persistent symptoms for months, if not years [3-9]. Pre-existing risk factors, peri-injury risk factors, and post-injury risk factors may all play a role in outcomes [10].

Often the mechanism of injury for a TBI in modern combat is related to a blast exposure. It is estimated that blast injuries from improvised explosive devices (IEDs) account for $52 \%$ to $80 \%$ of all casualties of the conflicts in Iraq and Afghanistan [11,12]. A blast injury is often associated with a primary high-velocity wave, as well as secondary or tertiary injuries such as flying debris or blunt impact when a body is thrown against a vehicle or the ground. Recent neuropsychological studies have found no measurable differences in cognitive performance between blast and blunt injuries $[13,14]$; however, it is unclear if effective treatment methods or recovery rates differ.

Service members serving in combat zones are at risk for multiple TBIs. Multiple TBIs can have a cumulative effect, present with more significant symptoms, and have slower recovery rates than a single concussion [15-19]. In extreme cases, chronic traumatic encephalopathy (CTE), a degenerative process that leads to dementia, has been posited to result from multiple mTBIs [20,21]. CTE symptoms include progressively severe memory and cognitive problems, depression, poor impulse control, suicidal ideations, and dementia [22-28]. It is unclear exactly how many concussions affect performance and could possibly trigger a degenerative process, as well as how these factors vary across individuals.

Combat-related cognitive dysfunction following a TBI may present differently than sports-related and non-combat TBI because service members often display symptoms that are co-morbid with post-traumatic stress disorder (PTSD) or mood changes [29-33]. It is difficult to determine the cause of cognitive symptoms in patients with co-occurring PTSD, and also to identify the most effective treatment for recovery of cognitive functions. For instance, problems with concentration and attention can result from TBI, but could be impacted further by sleep disturbances, medications, and internal distractions.

There is a need for novel evidence-based approaches for treating combat-related and non-combated-related cognitive symptoms of TBI, especially following mTBI [34]. Numerous reviews summarize the evidence for the effectiveness of traditional cognitive rehabilitation $[35,36]$, with some specific interventions showing moderate or strong evidence for efficacy [ 37 , $38,40,41,49]$. However, few studies focus on cognitive rehabilitation for TBI, and even fewer are specific to military TBI. Novel approaches to cognitive stimulation and rehabilitation have been introduced to the commercial market through computer-based tools over the past decade. This corresponds with the increased demand for self-paced, non-invasive options for both preventive mental wellness and cognitive rehabilitation. The new digital "brain health" market was estimated at over $\$ 1$ billion in 2012, and is expected to reach $\$ 6$ billion by 2020 [42]. 
Many commercially-available CBCT programs are intended to improve the functioning of the user's brain by using a neuroplasticity approach. CBCT exercises provide hundreds of repetitions in a given session. Previous research on these programs has shown overall positive results; however, most studies have been conducted on the general aging and pediatric populations. Studies conducted with the aging population demonstrate improvements in attention, memory, and other cognitive domains after using a structured CBCT program [43-48]. Generalization and carryover to improvements in daily activities have also been seen in the aging population for up to 5 years posttraining [49]. CBCT that targets auditory processing, working memory, and attention have shown effective results in school-aged children [50-52].

At the time this project was initiated, only a limited number of studies using CBCT programs with the TBI population had been published [53]. Introducing these products to the TBI and non-TBI population with cognitive symptoms at Walter Reed National Military Medical Center (WRNMMC) provided an opportunity to evaluate patient response and observe the feasibility of offering treatment with high-intensity dosing. Evidence for the effectiveness of such programs for both civilian and military patients with cognitive dysfunction would have implications for resiliency, telerehabilitation, and independent patient use in remote locations when one-on-one therapy is limited. Effective CBCT tools for enhancing rehabilitation could also serve to reduce costs of care and facilitate community re-entry.

In order to explore some of these technologies as a means for novel intervention, an interdisciplinary team at WRNMMC opened the first "Brain Fitness Center" (BFC) in 2008. In 2012, a satellite BFC was opened at Fort Belvoir Community Hospital (FBCH) to extend clinical opportunities and increase research recruitment. Identical CBCT programs were set up for clinical and research use at FBCH. Patients are administered the same outcome measures and all data are merged for research purposes.

The influx of emerging CBCT programs, demonstrated efficacy in other populations, and the large TBI population at the MTF, made WRNMMC an ideal setting for a "brain gym." The BFC provides recovering patients more access to cognitively stimulating exercise than traditional therapies alone. The gym concept was designed to allow patients the option to train independently and strengthen targeted cognitive goals established by their rehabilitation team. Adding CBCT to patients' treatment plans also allows for the continued use of the computer programs after being discharged to their homes where individual therapy may be unavailable due to location or cost. The BFC was not implemented to replace traditional cognitive rehabilitation. Instead, it was designed as an adjunct service providing complementary cognitive stimuli to enhance the rehabilitation process.

The BFC is located in an outpatient building at WRNMMC. WRNMMC is an MTF (a hospital, clinic, or medical center on a military base or post) comprising a full-service inpatient hospital and large outpatient diagnostic and rehabilitative clinics. When a service member is injured in combat, he or she is most often transported from the battlefield, usually through Germany, to WRNMMC. Services at WRNMMC range from immediate, intensive care to long hospital stays for patients with polytraumatic injuries. Once patients are discharged from an inpatient hospital stay, they may continue to live at WRNMMC and use the outpatient clinics for their medical care and rehabilitative services. The rehabilitation process, especially for those with limb amputations, can take months to years. Methods, dosing, and duration of cognitive and physical rehabilitation are varied and customized to the individual patient.

Patients participating in the BFC as part of or separate from their clinical rehabilitation, and those who are not enrolled in the research protocol, have the flexibility to use the BFC as little or as much as they like. Neuroplasticity-based research studies conducted with computer-based programs on other populations indicate the need for high-dose intensity to achieve both functional changes [54,55] and neuronal strengthening and rewiring [56-59]; therefore, patients using the $\mathrm{BFC}$ are encouraged to come at least two to three times per week for 6 to 8 weeks.

The computer programs selected for initial use in the BFC did not have a body of literature to support their use in this population, but were chosen based on experience in other patient populations or design factors inherent in the computer programs. In order to acquire outcome data, the staff in the BFC administers objective measures for all clinical BFC patients before, during, and after the use of a computer-training program in the BFC.

\section{Study goals}

This first study conducted in the BFC was designed to examine the effect of brain-training pro- 
grams for military service members returning from combat with symptoms of cognitive dysfunction via randomized assignment to one of two computerized cognitive rehabilitation programs or to a control group (Treatment as Usual). The objectives of the study were: (1) to measure and compare participants' compliance amongst the two computer programs using an attendance database; (2) to measure and compare aspects of cognitive improvement amongst the three groups using the Automated Neuropsychological Metrics (ANAM) [60]; and (3) to measure and compare participants' satisfaction and self-perception of improvement as measured by self-report questionnaires. This report addresses the first of these goals.

\section{Methods}

The protocol was designed as a randomized, controlled study to evaluate the first two CBCT programs purchased for use in the BFC, Dakim ${ }^{\circledR}$ Brain Fitness and Posit Science ${ }^{\circledR}$ Brain Fitness Classic.

\subsection{Participants}

Inclusion criteria required a diagnosis of a mild to moderate TBI sustained while serving in combat. Participants who had combat-related subjective cognitive complaints but did not meet the criteria of a TBI as determined by the standard DoD definition [61] were included in the non-TBI arm for additional exploratory analysis. Candidates were excluded if their cognitive symptoms were not associated with combat, if they were ever diagnosed with a severe or penetrating TBI, or if they sustained an additional TBI within 2 years prior to the most recent TBI. Other exclusion criteria were other medical, neurological, or psychological conditions that would interfere with participation, or a referral for a particular treatment that precluded the option of being randomized into a group.

Sample size was estimated based on the desired power for the main outcome measure across three groups of TBI patients using a repeated-measures analysis of variance (RM-ANOVA). To detect a difference of 10 between any two of the main groups on the MPAI total raw score (range 0 to 111 ), with $\alpha=0.05$ and power $=80 \%$, sample size targeted 69 participants with TBI (23 per group: Dakim Brain Fitness, Brain Fitness Classic, and control). To explore differences between non-TBI and TBI groups, enrollment targeted an additional 45 subjects (15 per group) who did not meet diagnostic criteria for TBI but reported cognitive dysfunction. Therefore, the total targeted sample size was 114.

\subsection{Groups}

Patients who consented to participate in the study were randomly assigned to one of three groups: Training Group A: Dakim Brain Fitness; Training Group B: Posit Science Brain Fitness Classic; or Control Group C: treatment-as-usual (TAU) without additional computer-based training. Both training groups also received TAU. After completing the study, all participants were instructed not to use CBCT programs or applications until after the 12-month follow-up phone call.

The Dakim Brain Fitness program was designed as a cognitive stimulation tool intended to improve concentration and memory in the elderly by warding off the effects of normal aging. Studies have indicated that high levels of participation in cognitively stimulating leisure activities are associated with a significantly reduced risk of dementia [62-66]. The Dakim program provides cognitively stimulating activities through entertaining, interactive, game-like cognitive exercises that span six cognitive domains: long-term memory, short-term memory, critical thinking, calculation, language, and visuo-spatial processing [67]. The program dynamically auto-adjusts within each cognitive domain throughout each session and over multiple sessions. A study using the Dakim program showed older adults performed better in standardized testing for delayed and immediate memory after 6 months [68].

Posit Science Brain Fitness Classic was also designed to attenuate cognitive decline in the normal, aging brain. The program targets auditory processing speed to enable users to process auditory information faster and more efficiently to improve overall thinking skills [69]. This program also adjusts to each user's cognitive performance to train at the appropriate level. Research studies have shown that the Posit Science program provides a mental exercise statistically superior to general mental activity, as measured by the extent to which older adults improve on cognitive tasks following training [70,71]. These changes were also reported to generalize to improvements in activities of daily living [72-74].

TAU at WRNMMC varies and is dependent on patient diagnosis, severity, time-post-onset of injury, and subjective complaints. Typical treatment includes behavioral health, physical therapy, occupational therapy, and speech-language pathology services.

\subsection{Assessments and training schedule}

All participants assigned to a CBCT group completed an initial evaluation, a post-treatment assess- 
ment, and were scheduled for a 12-month follow-up phone call. Outcomes were measured using a compliance tracker, a satisfaction survey, and subjective and objective performance assessments. Compliance was determined by the number of completed sessions and the overall duration of treatment. Preliminary results based on satisfaction and performance measures are being prepared for a separate report.

To complete the training, the participants were required to come to the BFC for one hour, five times per week to complete 30 computer sessions within 6 weeks. This intensity was chosen to closely match the recommended dosing (40 hours) of Posit Science's Brain Fitness Classic. The total number of sessions was reduced from 40 in 8 weeks to 30 in 6 weeks in anticipation of a typical minimum length-of-stay at WRNMMC. Each Posit Science session was designed to last approximately 40-60 minutes. Because the Dakim program is administered in 20-minute segments, participants were asked to complete two segments per session for 40 minutes of computer training each day. The BFC was designed as a structured, controlled environment supported by clinical and research staff. Research participants worked independently on the computer program; a research assistant was present for technical issues and documentation purposes only.

If a participant left the facility or choose to terminate participation prior to completing the 6-week program, that participant's data remained in the study to minimize bias. Discharge testing was completed whenever participants completed the 30 -session training or after their last session, if possible, if they dropped out earlier.

\section{Results}

\subsection{Participant recruitment and enrollment}

Figure 1 provides a flowchart of potential participants and study participation. A total of 291 patients were referred to the WRNMMC and FBCH BFCs for computer training over the 2.5 year recruitment period (August 2010-December 2012). Each patient was evaluated and screened for research eligibility. Of those 291,120 (41\%) were eligible to participate. Reasons for ineligibility were: non-combat-related injury or cognitive symptoms $(n=63)$; other medical, neurological, or psychiatric conditions $(n=46)$; severe or penetrating TBI $(n=30)$; referrals to the BFC for a specific computer program $(n=13)$; multiple TBIs

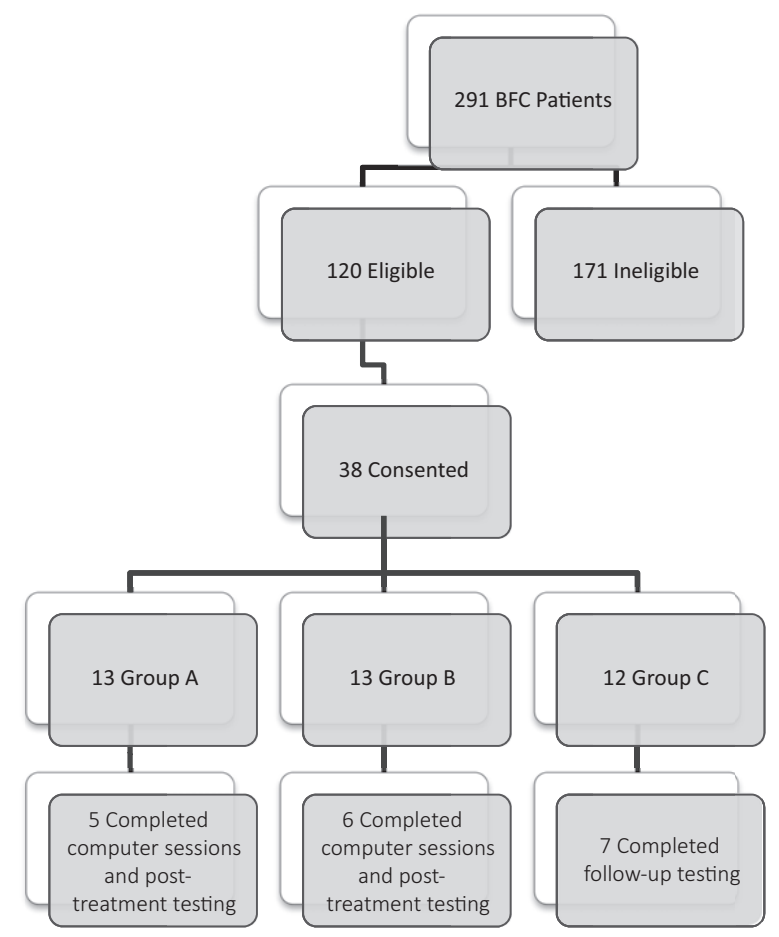

Fig. 1. Flowchart of participant enrollment and completion.

over a 2-year period ( $n=13$ ); and "other" including unclear TBI diagnosis $(n=6)$.

Of the 120 eligible research candidates, 38 (37 men, 1 woman) agreed to enroll in the study. Thirteen participants (11 TBI, 2 non-TBI) were assigned to Group A, 13 (10 TBI, 3 non-TBI) to Group B, and 12 (10 TBI, 2 non-TBI) to Group $\mathrm{C}$. The remaining 82 candidates $(68 \%)$ reported that they were uninterested in participating $(n=35)$; were not willing or able to commit to the required daily sessions either because of intensive rehabilitation requirements $(n=13)$ or because they were not living at WRNMMC or anticipated leaving WRNMMC soon to return home $(n=18)$; or because they did not want to be randomized to the control group $(n=16)$.

\subsection{Study compliance and completion}

Five participants in Group A and six participants in Group B completed all required computer sessions and the post-treatment evaluation (Fig. 2). Seven participants in Group C completed the follow-up evaluation. The 12-month follow up phone calls were canceled due to low retention.

Figure 2 illustrates the number of sessions and duration of treatment for each participant in Groups A and 


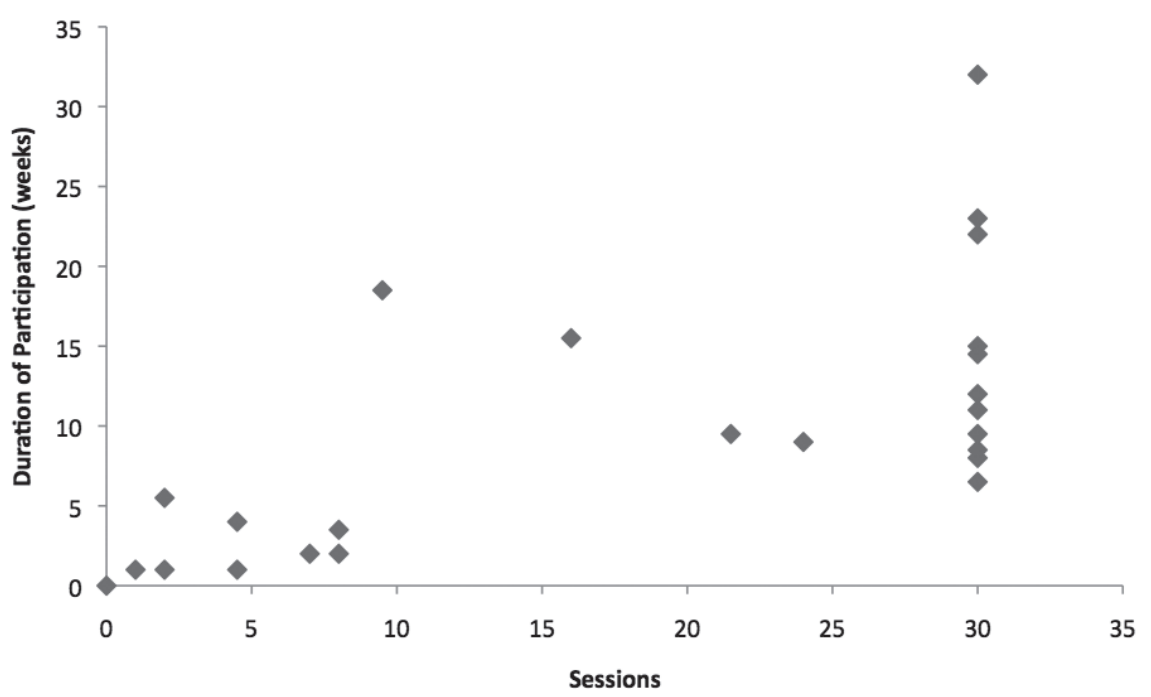

Fig. 2. Number of sessions completed and the duration of participation for each research participant in Groups A and B $(N=26)$. There are two data points at $(0,0)$ and $(30,8)$. Study design intended 30 sessions in 6 weeks.

B. Although 11 of the 26 participants completed the required number of sessions, none finished all 30 sessions in the prescribed 6 weeks, instead taking 6.5-32 weeks. Eleven of 15 participants $(73.3 \%)$ who dropped out of the study did so before completing one-third of the required sessions. There was no difference between computer groups in terms of compliance or drop-out rate.

Approximately 2.5 years after study recruitment began, the Posit Science Classic program was no longer commercially available as the company moved to a web-based platform. The content of the program changed and for research purposes could not be compared to the version used in the study. Due to the new platform in addition to low enrollment, the research team decided to terminate the study. Beyond recruitment and compliance, goals of this report were adjusted to focus on study challenges and to propose solutions for future research in this area.

\section{Study challenges and proposed solutions}

We are developing new research protocols as more innovative technology options become available. It is essential to evaluate the limitations of our first study to determine the implications for clinical research in the $\mathrm{BFC}$ moving forward. The remainder of this paper will outline challenges of the study and propose solutions. The challenges are divided into two categories 1) Recruitment and Eligibility Requirements, and 2) Participant Requirements and Dosing, as summarized in Tables 1 and 2 .

\subsection{Challenge 1: Recruitment and eligibility requirements}

\subsubsection{Initial recruitment plan \\ Challenge}

We anticipated that a sufficient number of eligible research candidates would be referred to the BFC from various clinical services. Although an adequate number of eligible patients were referred, $68 \%$ declined to participate in the study. With the realization that recruitment from WRNMMC alone was not realistic, a second site was added partway through the study but enrollment goals remained unattainable in the projected time period.

\section{Solution}

A more aggressive recruitment plan is needed. The second Fort Belvoir site provided a 30\% increase in patient referrals and $45 \%$ increase in enrollment. If other sites supplemented participant enrollment similarly, three additional sites would need to be added to achieve the target sample size over the next 3 years. Even with additional sites, more active recruitment outside of the two BFCs is also necessary. To maintain a military sample, additional MTF or Veterans Administration (VA) sites would be beneficial.

\subsubsection{Eligibility restricted to combat-related TBI Challenge}

The first of two eligibility requirements to present an unanticipated challenge was that all participants 
Table 1

Proposed solutions to address the challenges of recruitment and eligibility requirements for a current computer-based cognitive training study at a military treatment facility

\begin{tabular}{lll}
\hline Initial study & Proposed solution & Anticipated change in eligible subjects \\
\hline Recruitment through BFC referrals at two sites & Add additional study-sites & $45 \%$ increase per year, per site \\
Eligibility restricted to combat-related symptoms & Inclusion of non-combat-related symptoms & $21.9 \%$ \\
Eligibility restricted to service members with no & Inclusion of service members with multiple & $4.7 \%$ \\
TBI within 2 years of most recent TBI & TBIs within 2 years of most recent TBI & \\
\hline
\end{tabular}

Table 2

Proposed solutions to the challenges of participant requirements and dosing for a feasibility study at a military treatment facility

\begin{tabular}{ll}
\hline Pilot study requirements & Proposed solution \\
\hline Sessions must be completed in the BFC & Web-based tools \\
Daily session/5x per week & Flexible scheduling with an option of only 1 weekly session in clinic \\
Group C (Treatment as Usual) & Sham or waitlist control group \\
12-month follow-up phone call & Revise 12-mo follow up for shorter time period and/or maintenance survey \\
No tangible incentive & Financial compensation for participation \\
\hline
\end{tabular}

must have combat-related symptoms. As previously discussed, a TBI incurred in combat is unique in terms of mechanism of injury and the combination of additional psychological symptoms. Thus, patients who acquired their injuries in a war zone may present differently, recover at different rates, and may respond uniquely to CBCT than patients whose injuries are acquired in a non-combat-related incident. Although WRNMMC is an MTF, we overestimated the number of patients who suffered a TBI in combat who would be able and willing to participate.

\section{Solution}

A solution to this challenge would be to allow service members with non-combat-related injuries to be included in the study. In the current study, this approach would have increased the percentage of eligible patients from $41 \%$ to $62.9 \%$.

\subsubsection{Eligibility restriction for number of TBI Challenge}

The second eligibility requirement to present a recruitment challenge was the lack of an additional TBI within two years prior to the recent TBI because multiple head injuries can have a cumulative effect. Given that the participant pool for this study is military service members returning from combat where they are often exposed to multiple blasts, some candidates reported more than one concussive episode.

\section{Solution}

A solution would be to allow participants who had sustained more than one TBI. Approximately 5\% more BFC patients would have been eligible for this study if multiple injuries were allowable for inclusion.

\subsection{Challenge 2: Participation requirements and dosing}

\subsubsection{Training Sessions in BFC \\ Challenge}

We anticipated that most participants would be living on the military base and their length of stay at WRNMMC would allow for a 6-week training course in the BFC. Busy rehabilitation schedules and community re-entry, either locally or remotely, prevented many participants from coming to the $\mathrm{BFC}$ on a regular basis. When this study was designed, most CBCT programs needed to be associated with a single computer, and data could not be transferred to another computer between visits. Technological limitations restricted our ability to allow the participants flexibility to complete sessions outside the BFC.

\section{Solution}

Since this study was designed, many more webbased options for CBCT have become available. Sitespecific training may no longer be necessary and an option for at-home use could be implemented. Web-based brain-training programs can be utilized anywhere a participant has access to a computer and the Internet. If needed, this could be accomplished with loaner laptops. A recent pilot study assessed the feasibility of individuals with TBI using a CBCT program unsupervised at home with only phone and email support. Compliance and completion rates revealed that independent use of a CBCT program with remote support is a viable option for this population [75].

\subsubsection{Recommended daily dosing Challenge}

The frequency and dosing of treatment was based on literature available at the time this study was de- 
signed. There is no gold standard for dosing recommendations; five visits per week proved to be an unrealistic expectation for this population in this setting. As seen in Fig. 2, 42\% of participants stopped coming to the BFC for training sessions before visit 10. Interestingly, $80 \%$ of those who attended the program for 10 visits remained and completed the remaining 20 visits. Unfortunately, conforming to participants' schedules may not be effective because of inadequate dosing. Offering a program with one to two visits per week might solve the problem of attrition, but it is unlikely to lead to the neuroplasticity required to improve learning. In addition, participants from a MTF may drop out of a long-term training program because they may be discharged to home or another treatment facility sooner than expected.

\section{Solution}

A potential solution to this challenge would be to require on-site training sessions once per week and offsite (web-based) training sessions four days per week. Participants who are self-directed and motivated may be able to work off-site for several weeks with only occasional check-in sessions. Another option is to offer a more intense program, such as two sessions per day for 2.5 weeks. The efficacy of alternate schedules would need to be established. Having multiple options for the MTF setting may help compliance and completion, but would increase the complexity of the study design.

\subsubsection{Treatment as usual control group Challenge}

There is high face-validity for coming to the BFC and using a CBCT. The commercialization of CBCT programs such as radio, television, and online advertisements has expanded over the past few years and service members are exposed to marketing outside WRNMMC. In addition, over the 2.5 years of study enrollment, the BFC went from a novel and modestly-used clinic at WRNMMC to a more standard option for rehabilitative intervention. If randomized to the control group, a participant was not permitted to use the BFC resources for the duration of the study and for one year following the final session. Although there was no research to support these programs for this population at the time this study was initiated, some candidates perceived that a valuable service would be withheld or denied if they were randomized to the control group. That is, they assumed that the active intervention arm was superior to TAU, and nearly $20 \%$ of them did not want to risk randomization to the control arm.

\section{Solution}

A more effective option for the control condition would be a placebo or sham computer program. Alternately, participants could be placed on a waiting list and allowed to use the BFC thereafter.

\subsubsection{Follow-up assessment \\ Challenge}

Participants were asked not to use CBCT tools for 12 months after their last session in the BFC. A 12-month follow-up phone call was intended to assess generalization and long-term effects of the 6-week CBCT. We planned to readminister each symptom self-report survey completed at baseline and after the 6-week training/control program during the follow-up call. Only a limited number of follow-up calls $(n=2)$ were completed at the time the decision was made to close the study to enrollment. Before being discharged, multiple participants reported that they wanted to continue the use of a CBCT program, highlighting the challenge of controlling out-of-clinic behaviors. Due to this concern combined with the low number of subjects who completed the study, the 12-month call was eventually abandoned.

\section{Solution}

The 12-month phone call should be revised to allow for multiple shorter-term follow-up calls and the examination of naturally continued use of CBCT programs. The commercial expansion and marketing of CBCT applications, and the real or perceived benefits indicated by users, make it unrealistic to expect this population to refrain from use for 12 months. It would be interesting to explore short- and long-term benefits of treatment, but it may be more important to determine whether participants independently choose to continue using the treatment program or a different brain-training tool.

\subsubsection{No tangible incentive Challenge}

Candidates for the study are informed at the time of consent that they may receive no direct benefit from participating in this study. No tangible incentive, reward, or financial compensation was offered for completion of this study. At the conception of this protocol, financial compensation was not allowed for military service members to participate in a study. Recently the WRNMMC Institutional Review Board made a decision to allow compensation for research participants. 


\section{Solution}

Considering the time commitment for this study, a monetary incentive is appropriate and may improve recruitment and retention.

\section{Discussion}

Advances in technology will continue to provide novel rehabilitation options. Determining the effectiveness of new products will rely on successful evidencebased research. The first study at WRNMMC and FBCH to conduct a randomized-controlled study assessing improvement in cognitive function and symptom self-report using CBCT programs fell short of recruitment and compliance goals. Although the study was fundamentally well-designed, an analysis of eligibility requirements, recruitment techniques, patient feedback, and compliance rates revealed challenges that are critical to resolve for the successful implementation of future research studies.

The MTF setting appears in many ways to be ideal for an intensive cognitive-training program. Many patients remain on site for an extended period of time and are seeking additional activities to supplement their routines. However, the patients' concentrated medical and psychological health care needs, and possibly lack of interest, affected enrollment and compliance efforts. The challenge is to create well-designed studies to determine treatment efficacy while conforming to the unique needs of military service members recovering from combat injuries.

The current study was initiated at the same time the BFC began accepting clinical patients, but only patients who met stringent inclusion criteria were recruited. Rather than restrict enrollment to service members with combat-related cognitive symptoms, widening eligibility requirements to more closely reflect our overall patient population would have been a more productive approach. Performance of combat vs. non-combat-related injuries and the number of TBIs or blast exposures could be examined as additional variables in future analyses. Also helpful would have been the inclusion of additional sites so that similar studies could be offered and monitored at polytrauma rehabilitation settings or VA facilities that are close to participants' homes. In addition, including civilian sites would allow comparisons between civilian and military populations.

A substantial number of eligible patients declined to participate in this study. A primary reason was the time commitment required to complete this study. Without any tangible incentives, participants are expected to dedicate 32 hours of evaluation and training in the BFC within a 6 -week time frame. The dosing requirement, flexibility of treatment locations, and incentives need to be reevaluated as new research protocols are developed.

High-dosage treatment not only affected enrollment, but also appeared to negatively affect compliance. Adhering to the recommended treatment intensity is considered essential for improving performance based on supporting literature on neuroplasticity. However, dosing flexibility and training location need to be revised to increase compliance. Fortunately, technological advances that allow remote access to the training programs offer a solution to this problem.

The BFC at WRNMMC is currently participating in, and will continue to develop, randomized controlled clinical treatment studies utilizing the proposed solutions learned from this first feasibility study. In addition, the research team is developing a database that includes attendance and performance data for all BFC users, which to date numbers nearly 500 health-care beneficiaries, including active-duty or retired service members and their dependents. Exploration of these clinical data will allow us to examine factors such as personal characteristics, practice dosage, and program selection that best predict cognitive improvement and functional outcomes.

\section{Conclusion}

Randomized controlled studies for cognitive rehabilitation in MTFs for patients injured in combat are needed. Our experience indicates that the population, setting, and dosing required for functional improvements to accrue pose many challenges. Successful implementation of a high-dose CBCT clinical trial in a military health-care environment will benefit from design features such as expanded inclusion criteria, sham or wait-list control options, clearly defined dosing flexibility, incentives, expanded study sites, and alternate training modalities afforded by changing technology.

\section{Acknowledgements}

This research was sponsored and funded through the Center for Neuroscience and Regenerative Medicine (CNRM), and the Defense and Veterans Brain Injury 
Center (DVBIC). The authors would like to thank Laura Sharkey, Gabriella Green-Oakes, and Wendy A. Law for their contributions to this research project and publication.

\section{Disclaimer}

The views expressed in this article are those of the authors and do not necessarily reflect the official policy or position of the Department of the Navy, Army, or Air Force, the Department of Defense, nor the U.S. Government.

\section{References}

[1] Okie S. Reconstructing lives-a tale of two soldiers. N Engl J Med. 2006; 355(25): 2609.

[2] Congressional Research Service. U.S. Military Casualty Statistics: Operation New Dawn, Operation Iraqi Freedom, and Operation Enduring Freedom. (RS22452, 05 February 2013), By Hannah Fischer. Accessed: May 17, 2013.

[3] Schretlen DJ, Shapiro AM. A quantitative review of the effects of traumatic brain injury on cognitive functioning. International Review of Psychiatry. 2003; 15: 341-349.

[4] Roe C, Sveen U, Alvsaker K, Bautz-Holter E. Postconcussion symptoms after mild traumatic brain injury: influence of demographic factors and injury severity in a 1-year cohort study. Disability and Rehabilitation. 2009; 31: 12351243.

[5] Dikmen, S, Machamer J, Fann JR, Temkin NR. Rates of symptom reporting following traumatic brain injury. Journal of the International Neuropsychological Society. 2010; 16: 401-411.

[6] Ruff RM, Camenzuli L, Mueller J. Miserable minority: emotional risk factors that influence the outcome of a mild traumatic brain injury. Brain Injury. 1996; 10(8): 551-566.

[7] Hoge CW, Goldberg HM, Castro CA. Care of war veterans with mild traumatic brain injury - flawed perspectives. N Engl J Med. 2009; 360(16): 1588-91.

[8] Schneiderman, AI, Braver ER, Kang HK. Understanding sequelae of injury mechanisms and mild traumatic brain injury incurred during the conflicts in Iraq and Afghanistan: persistent postconcussive symptoms and posttraumatic stress disorder. Am J Epidemiol. 2008; 167: 1446-1452.

[9] Iverson GL, Zasler ND, Lange RT. Post-concussive disorder. In Brain injury medicine, N.D. Zasler, D.I. Katz, R.D. Zafonte, eds, New York: Demos Medical Publishing, 2007, pp. 373-405.

[10] Helmick K. Cognitive rehabilitation for military personnel with mild traumatic brain injury and chronic postconcussional disorder: Results of April 2009 consensus conference. NeuroRehabilitation. 2010; 26(3): 239-255.

[11] Owens BD, Kragh JF Jr., Wenke JC, Macaitis J, Wade CE, Holcomb JB. Combat wounds in operation Iraqi Freedom and operation Enduring Freedom. J. Trauma 2008; 64: 295-299.

[12] Galaraneau MR, Woodruff SI, Dye JL, Mohrle CR, Wade AL. Traumatic brain injury during Operation Iraqi Freedom: findings from the United States Navy - Marine Corps Combat Trauma Registry. J Neurosurgery. 2009; 108: 950-57.
[13] Lange RT, Pancholi S, Brickell TA, Sakura S, Bhagwat A, Merritt V, et al. Neuropsychological Outcome from blast versus non-blast: Mild traumatic brain injury in U.S. Military Service Members. J Int Neuropsychology Soc. 2012; 30: 111.

[14] Cooper DB, Chau PM, Armistead-Jehle P, Vanderploeg RD, Bowles AO. Relationship between mechanism of injury and neurocognitive functioning in OEF/OIF service members with mild traumatic brain injuries. Mil Med. 2012; 177(10): 115760.

[15] Grossman EJ, Ge Y, Jensen JH, Babb JS, Miles L, Reaume J, et al. Thalamus and cognitive impairment in mild traumatic brain injury: A Diffusional Kurtosis Imaging Study. J Neurotrauma. 2012; 29(13): 2318-27.

[16] Henry LC, Tremblay J, Tremblay S, Lee A, Brun C, Lepore N, et al. Acute and chronic changes in diffusivity measures after sports concussion. J Neurotrauma. 2011; 28(10): 2049-59.

[17] Castile L, Collins CL, McIlvain NM, Comstock RD. The epidemiology of new versus recurrent sports concussions among high school athletes, 2005-2010. Br J Sports Med. 2012; 46(8): 603-10.

[18] Guskiewicz KM, McCrea M, Marshall SW, Cantu RC, Randolph C, Barr W, et al. Cumulative effects associated with recurrent concussion in collegiate football players: The NCAA concussion study. JAMA. 2003; 290: 2549-2555.

[19] MacGregor AJ, Dougherty AL, Morrison RH, Quinn KH, Galarneau MR. Repeated concussion among U.S. Military personnel during Operation Iraqi Freedom. Journal of Rehabilitation Research \& Development. 2011; 48(10): 12691277.

[20] McKee AC, Cantu RC, Nowinski CJ, Hedley-Whyte ET, Gavett BE, Budson AE, et al. Chronic traumatic encephalopathy in athletes: Progressive tauopathy after repetitive head injury. J Neuropathol Exp Neurol. 2009; 68: 709-735.

21] Gavett BE, Stern RA, McKee AC. Chronic traumatic encephalopathy: A potential late effect of sport-related concussive and subconcussive head trauma. Clin Sports Med. 2011; 30: 179-188, xi.

[22] Miller H. Mental after-effects of head injury. Proc R Soc Med. 1966; 59: 257-261.

[23] Stern RA, Riley DO, Daneshvar DH, Nowinski CJ, Cantu $\mathrm{RC}, \mathrm{McKee} \mathrm{AC}$. Long-term Consequences of repetitive brain trauma: Chronic traumatic encephalopathy. PM\&R. 2011; 3: S460-S467.

[24] Stern RA, Andersen SL, Gavett BE. Executive functioning in dementia. In: Kowall NW, Budson AE, eds. The Handbook of Alzheimer's Disease and Other Dementias. Chichester, West Sussex, UK: Wiley-Blackwell, 2011.

[25] Hall RC, Chapman MJ. Definition, diagnosis, and forensic implications of postconcussional syndrome. Psychosomatics. 2005; 46: 195-202.

[26] Giza CC, Hovda DA. The neurometabolic cascade of concussion. J Athl Train. 2001; 36: 228-235.

[27] Sojka P, Stalnacke BM, Bjornstig U, Karlsson K. One-year follow-up of patients with mild traumatic brain injury: Occurrence of post-traumatic stress-related symptoms at follow-up and serum levels of cortisol, S-100B and neuron-specific enolase in acute phase. Brain Inj. 2006; 20: 613-620.

[28] Sterr A, Herron KA, Hayward C, Montaldi D. Are mild head injuries as mild as we think? Neurobehavioral concomitants of chronic postconcussion syndrome. BMC Neurol. 2006; 6 : 7.

[29] Hoge CW, Mcgurk D, Thomas J L, Cox AL, Engel CC, Cas- 
tro CA. Mild traumatic brain injury in U.S. Soldiers returning from Iraq. N. Engl. J. Med. 2008; 358: 453-463.

[30] Levin HS, Wilde E, Troyanskaya M, Peterson NJ, Scheibel $\mathrm{R}$, Newsome M, et al. Diffusion tensor imaging of mild to moderate blast-related traumatic brain injury and its sequelae. J of Neurotrauma. 2010; 27(4): 683-694.

[31] Rosenfeld JV, Ford NL. Bomb blast, mild traumatic brain injury and psychiatric morbidity: A review. Injury. 2010; 41: 437-443.

[32] Forbes D, Creamer M, Bisson JI, Cohen JA, Crow BE, Foa $\mathrm{EB}$, et al. A guide to guidelines for the treatment of PTSD and related conditions. Journal of Traumatic Stress. 2010; 23: 537-52.

[33] Jaffee MS, Meyer KS. A brief overview of traumatic brain injury (TBI) and post-traumatic stress disorder (PTSD) within the Department of Defense. Clin. Neuropsychol. 2009; 23: 1291-1298.

[34] Von Ah D, Carpenter JS, Saykin A, Monahan P, Wu J, Yu M, et al. Advanced cognitive training for breast cancer survivors: A randomized controlled trial. Breast Cancer Res Treat. 2012; 135(3): 799-809.

[35] Cicerone KD, Dahlberg C, Malec JF, Langenbahn DM, Felicetti T, Kneipp S, et al. Evidence-based cognitive rehabilitation: updated review of the literature from 1998 through 2002. Archives of Physical Medicine and Rehabilitation. 2005; 86(8): 1681-1692.

[36] Cappa SF, Benke T, Clarke S, Rossi B, Stemmer B, Heugten $\mathrm{CM}$. EFNS guidelines on cognitive rehabilitation: report of an EFNS task force. European Journal of Neurology. 2005; 12(9): 665-680.

[37] Sohlberg MM, Avery JA, Kennedy M, Ylvisaker M, Coelho C, Turkstra L, et al. Practice guidelines for direct attention training. Journal of Medical Speech-Language Pathology. 2003; 11(3): xix-xxxix

[38] Kaschel R, Della Sala S, Cantagallo A, Fahlböck A, Laaksonen R, Kazen M. Imagery mnemonics for the rehabilitation of memory: A randomized group controlled trial. Neuropsychological Rehabilitation. 2002; 12(2): 127-153.

[39] Sohlberg MM, Kennedy M, Avery J, Coelho C, Turkstra L, Ylvisaker M, et al. Evidence-based practice for the use of external aids as a memory compensation technique. Journal of Medical Speech Language Pathology. 2007; 15(1): xv-li.

[40] Dahlberg CA, Cusick CP, Hawley LA, Newman JK, Morey CE, Harrison-Felix CL, et al. Treatment efficacy of social communication skills training after traumatic brain injury: A randomized treatment and deferred treatment controlled trial. Archives of Physical Medicine and Rehabilitation. 2007; 88(12): 1561-1573

[41] Kennedy MR, Coelho C, Turkstra L, Ylvisaker M, Sohlberg $\mathrm{MK}$, Yorkston K, et al. Intervention for executive functions after traumatic brain injury: A systematic review, meta-analysis and clinical recommendations. Neuropsychological Rehabilitation. 2008; 18(3): 257-299.

[42] Fernandez A. Transforming Health and digital Tools to assess, monitor and enhance cognition across the lifespan; A digital health market 2012-2020. Sharpbrains 2012.

[43] Galante E, Venturini G, Fiaccadori C. Computer-based cognitive intervention for dementia: preliminary results of a randomized controlled trial. Giornale italiano di medicina del lavoro ed ergonomia. 2007; 29: B26-32.

[44] Brehmer Y, Westerberg H, Backman L. Working-memory training in younger and older adults: training gains, transfer, and maintenance. Frontiers in Human Neuroscience. 2012; 6(63): $1-7$
[45] Hardy JL, Drescher D, Sarkar K, Kellett G, Scanlon M. Enhancing visual attention and working memory with a webbased cognitive training program. Mensa Research Journal. 2011; 42(2): 13-20.

[46] Verghese J, LeValley A, Derby C, Kuslansky G, Katz M, Hall $\mathrm{C}$, et al. Leisure activities and the risk of amnestic mild cognitive impairment in the elderly. Neurology. 2006; 66(6): 821827.

[47] Wilson RS, Scherr PA, Schneider JA, Tang Y, Bennett DA. Relation of cognitive activity to risk of developing Alzheimer's disease. Neurology. 2007; 69(20): 1911-20.

[48] Finn M, McDonald S. Computerized cognitive training for older persons with mild cognitive impairment: A pilot study using a randomized controlled trial design. Brain Impairment. 2011; 12(3): 187-199.

[49] Willis SL, Tennstedt SL, Marsiske M, Ball K, Elias J, Koepke $\mathrm{KM}$, et al. Long-term effects of cognitive training on everyday functional outcomes in older adults. Journal of the American Medical Association. 2006; 296(23): 2805-2814.

[50] Gray SA, Chaban P, Martinussen R, Goldberg R, Gotlieb H, Kronitz R, et al. Effects of a computerized working memory training program on working memory, attention, and academics in adolescents with severe LD and comorbid ADHD: A randomized controlled trial. J Child Psychology Psychiatry. 2012; 53(12): 1277-84.

[51] Alexander DW, Frost B P. Decelerated synthesized speech as a means of shaping speed of auditory processing of children with delayed language. Perceptual \& Motor Skills. 1982; 55(3, Pt 1): 783-792.

[52] Klingberg T, Fernell E, Olesen PJ, Johnson M, Gustafsson P, Dahlstrom K, et al. Computerized training of working memory in children with ADHD- A randomized, controlled trial. J. American Academy of Child and Adolescent Psychiatry. 2005; 44(2): 177-186.

[53] Chen SHA, Thomas JD, Glueckauf RL, Bracy OL. The effectiveness of computer-assisted cognitive rehabilitation for persons with traumatic brain injury. Brain Injury. 1997; 11: 197209.

[54] Fisher M, Holland C, Subramaniam K, Vinogradov S. Neuroplasticity-based cognitive training in schizophrenia: An interim report on the effects 6 months later. Schizophr Bull. 2010; 36(4): 869-879.

[55] NIH Consensus Development Panel on Rehabilitation of Persons with Traumatic Brain Injury. Rehabilitation of persons with traumatic brain injury. JAMA. 1999; 282(10): 974.

[56] Fisher M, Holland C, Merzenich MM, Vinogradov S. Using neuroplasticity-based auditory training to improve verbal memory in schizophrenia. Am J Psychiatry. 2009; 166(7): 805-11.

[57] Merzenic MM. Cortical plasticity contributing to child development. In: Mechanisms of cognitive development: Behavioral and neural perspectives. Psychology Press. 2001; 67-95.

[58] Buonomano DV, Merzenich MM. Cortical plasticity: from synapses to maps. Annual Review of Neuroscience. 1998; 21(1): 149-186.

[59] Berry AS, Zanto TP, Clapp WC, Hardy JL, Delahunt PB, Mahncke HW, et al. The influence of perceptual training on working memory in older adults. PLoS ONE. 2010; 5(7).

[60] Johnson DR, Vincent AS, Johnson AE, Gilliland K, Schlegel RE. Reliability and construct validity of the Automated Neuropsychological Assessment Metrics (ANAM) mood scale. Archives of Clinical Neuropsychology. 2008; 23(1): 73-85.

[61] Department of Veteran Affairs, Department of Defense. Washington, DC: The Management of Concussion/mTBI 
Working Group.VA/DoD Clinical Practice Guideline for the Management of Concussion/Mild Traumatic Brain Injury (mTBI): Guideline Summary, 2009.

[62] Akbaraly TN, Portet F, Fustioni S, Dartiques JF, Artero S, Rouaud $\mathrm{O}$, et al. Leisure activities and the risk of dementia in the elderly: The Three-City study. Leisure activities and the risk of dementia in the elderly. Neurology. 2009; 73: 854-861.

[63] Fritsch T, Smyth KA, Debanne SM, Petot GJ, Friedland RP. Participation in novelty-seeking leisure activities and Alzheimer's disease. Journal of Geriatric Psychiatry and Neurology. 2005; 18: 134-141.

[64] Hall CB, Lipton RB, Sliwinski M, Katz MJ, Derby CA, Verghese J. Cognitive activities delay onset of memory decline in persons who develop dementia. Neurology. 2009; 73: 356-361.

[65] Verghese J, Lipton RB, Katz MJ, Hall CB, Derby CA, Kuslanksy $\mathrm{G}$, et al. Leisure activities and the risk of dementia in the elderly. The New England Journal of Medicine. 2003; 348: 2508-2516.

[66] Willis SL, Tennstedt SL, Marsiske M, Ball K, Elias J, Koepke $\mathrm{KM}$, et al. Long-term effects of cognitive training on everyday functional outcomes in older adults. Journal of the American Medical Association. 2006; 296: 2805-2814.

[67] Dakim Brain Fitness [homepage on the Internet]. California: Dakim Inc; 2013 [Updated 2013; cited 2013 Feb 01]. Available from: http://www.dakim.com.

[68] Miller KJ, Dye RV, Kim J, Jennings JL, O'Toole E, Wong $\mathrm{J}$, et al. Effect of a computerized brain exercise program on cognitive performance in older adults. American Journal of Geriatric Psychiatry. 2013; 21(7): 655-63.
[69] PositScience [homepage on the Internet]. California: PositScience Inc; 2013 [updated 2012 June 06; cited 2013 Feb 01]. Available from: http://positscience.com.

[70] Smith GE, Housen P, Yaffe K, Ruff R, Kennison RF, Mahncke $\mathrm{HW}$, et al. A cognitive training program based on principles of brain plasticity: results from the improvement in memory with plasticity-based adaptive cognitive training (IMPACT) study. Journal of the American Geriatrics Society. 2009; 57(4): 594603.

[71] Edwards J, Wadley V, Myers Re, Roenker DL, Cissell G, Ball $\mathrm{K}$. Transfer of a speed of processing intervention to near and far cognitive functions. Gerontology. 2002; 48(5): 329-40.

[72] Lebowitz M, Cantor J, Gordon W, Spielman L, Ashman T, Tsaousides T, et al. Examining the usability of a computerized cognitive training program in people with traumatic brain injury: A pilot study. Archives of Physical Medicine and Rehabilitation. 2009; 90(10): e18-e19.

[73] Lundqvist A, Grundström K, Samuelsson K, Rönnberg J. Computerized training of working memory in a group of patients suffering from acquired brain injury. Brain Inj. 2010; 24(10): 1173-83

[74] Ball K, Edwards JD, Ross LA. The impact of speed of processing training on cognitive and everyday functions. Journals of Gerontology. 2007; 62B (Special Issue 1): 19-31.

[75] Lebowitz M, Dams-O'Connor K, Cantor J. Feasibility of computerized brain plasticity-based cognitive training after traumatic brain injury. Journal of Rehabilitation Research and Development. 2012; 49: 1547-1556. 